\title{
Decreased expression and clinical significance of miR-148a in hepatocellular carcinoma tissues
}

\author{
Linjiang Pan ${ }^{1 \dagger}$, Suning Huang ${ }^{1+}$, Rongquan $\mathrm{He}^{2}$, Minhua Rong ${ }^{3}$, Yiwu Dang ${ }^{4}$ and Gang Chen ${ }^{4 *}$
}

\begin{abstract}
Background: Aberrant expression of microRNA-148a (miR-148a) has been reported in several types of malignancies. However, its expression and clinicopathological significance in hepatocellular carcinoma (HCC) has not been entirely clarified. Our objective was to investigate the clinicopathological contribution of the miR-148a expression in HCC formalin-fixed paraffin-embedded (FFPE) tissues.

Methods: Eighty-nine HCC and their para-cancerous liver tissues were recruited. Total mRNA including miRNA was isolated and miR-148a expression was determined by using real time RT-qPCR. Furthermore, the relationship between the miR-148a level and clinicopathological features was explored.
\end{abstract}

Results: Significantly lower miR-148a expression in HCC tissues was observed than that in adjacent noncancerous hepatic tissues. miR-148a expression was also correlated to clinical TNM stage, metastasis, status of capsular infiltration and numbers of tumor nodes.

Conclusions: Underexpression of miR-148a might be associated with HCC tumorigenesis and deterioration of HCC. miR-148a might act as a suppressor miRNA of HCC and it therefore has a potential role in prognosis of HCC patients.

Keywords: miR-148a, Hepatocellular carcinoma, Metastasis, Paraffin-embedded tissues, RT-qPCR

\section{Background}

Hepatocellular carcinoma (HCC) is ranked as the sixth most frequent cancer and the third leading cause of cancer-related deaths globally [1,2]. In 2012, 782,000 new cases and 746,000 deaths from HCC occurred in the whole world. The burden of the disease is the highest in Eastern Asia, sub-Saharan Africa, and Melanesia with the endemic infection of hepatitis B virus (HBV). In the meantime, in Japan, United States, and Europe, hepatitis $\mathrm{C}$ virus (HCV) infection is predominant, and consequently, it has become the key risk factor for contracting HCC in these regions [3-7]. Although the improvement of molecular biology has led to the identification of new tumor markers that play vital roles in the treatment and prognosis of $\mathrm{HCC}$, more tumor markers are still required for effective early diagnosis and monitoring of the curative effect of HCC [8-15].

\footnotetext{
*Correspondence: chen_gang_triones@163.com

${ }^{\dagger}$ Equal contributors

${ }^{4}$ Department of Pathology, First Affiliated Hospital of Guangxi Medical University, 6 Shuangyong Road, Nanning, Guangxi Zhuang Autonomous Region 530021, PR China

Full list of author information is available at the end of the article
}

MicroRNAs, a major class of small non-coding RNAs, are well-conserved very small RNA molecules (20 to 22 nucleotides) that can negatively modulate gene expression post-transcriptionally. Accumulating evidence over the past decades highlights the significance of microRNAs as vital regulators of many essential physiological processes such as cell proliferation, differentiation, apoptosis, and embryonic development [16]. Dysregulation of microRNA expression has also been inferred in many diseases as well as in cancers [17-21]. Extensive profiling studies over recent years have shown that a variety of miRNAs are abnormally expressed in HCC [22,23]. Among all the HCC-related miRNAs, miR-148a has been reported to be significantly reduced in HCC tissues, compared with normal livers $[24,25]$. However, the relationship between miR-148a level and the clinicopathological parameters of HCC remains obscure. In the current study, we therefore investigated the expression of miRNA148a in HCC and their matched adjacent noncancerous liver tissues in formalin-fixed paraffin-embedded (FFPE) surgically resected samples. Furthermore, we studied the 
relationship between miR-148a expression and clinicopathological characteristics of HCC.

\section{Methods}

\section{Tissue samples}

This retrospective study included 89 cases of HCCs and their paired paraneoplastic liver FFPE tissues. The age of the HCC patients ranged from 29 to 82 years old, with a mean age of 52 years. Clinicopathological information extracted from medical records has been summarized in Table 1. Adjacent noncancerous liver tissues were at least $2 \mathrm{~cm}$ away from the tumor node. All cases were initial hepatectomies without treatment and from hepatectomies performed in the First Affiliated Hospital of Guangxi Medical University, PR China between March 2010 and December 2011. The study protocol was approved by the Ethical Committee of the First Affiliated Hospital of Guangxi Medical University. Written informed consent was obtained from the patients and clinicians for the usage of the samples for research. All samples were reviewed and diagnosed by two independent pathologists.

\section{RT-qPCR}

Total RNA including miRNA was isolated from tumor sections using the miRNeasy FFPE Kit (QIAGEN, KJ Venlo, Netherlands) according to our previous reports [26-28]. RNA concentrations were determined by Nanodrop 2000 (Wilmington, DE, USA). A combination of RUN6B and RUN48 was the housekeeping genes for detection of miR-148a expression $[27,28]$. The primers for miR-148a, RNU6B and RNU48 were included in TaqMan MicroRNA Assays (4427975, Applied Biosystems, Life Technologies Grand Island, NY, USA). The reverse primers were also used for reverse transcription with TaqMan ${ }^{\circ}$ MicroRNA Reverse Transcription Kit (4366596, Applied Biosystems, Life Technologies Grand Island, NY, USA) in a total volume of $10 \mu \mathrm{l}$. Real time RTqPCR for miRNA was performed with Applied Biosystems PCR7900. The miR-148a abundance in each sample was normalized to its references. The expression of miR$148 \mathrm{a}$ in the FFPE experiments was calculated with the formula $2^{-\Delta \mathrm{cq}}[26-29]$.

\section{Statistical analysis}

SPSS 20.0 (Munich, Germany) was performed for statistical analysis. Results were representative of three independent experiments. Values were presented as the mean \pm standard deviation (SD). Student's paired or unpaired $t$-test was used to analyze significance between paired or unpaired groups. One-way analysis of variance (ANOVA) test was used to analyze significance between groups of various differentiations. Correlations were calculated by Spearman's method. A receiver operator
Table 1 Relationship between the expression of miR-148a and clinicopathological features in hepatocellular carcinoma (HCC) $(\overline{\boldsymbol{x}} \pm \mathbf{s})$

\begin{tabular}{|c|c|c|c|c|c|}
\hline \multicolumn{2}{|c|}{ Clinicopathological features } & \multirow[t]{2}{*}{$\mathrm{n}$} & \multicolumn{3}{|c|}{ MiR-148a relevant expression } \\
\hline & & & $2^{-\triangle c q}$ & $t$ & $P$ \\
\hline \multirow[t]{2}{*}{ Tissue } & $\mathrm{HCC}$ & 89 & $0.87 \pm 0.50$ & $-7.762 \Delta$ & $<0.00$ \\
\hline & $\begin{array}{l}\text { Adjacent } \\
\text { noncancerous } \\
\text { liver }\end{array}$ & 89 & $1.44 \pm 0.61$ & & \\
\hline \multirow[t]{2}{*}{ Age } & $\geq 50$ & 43 & $0.83 \pm 0.49$ & -0.727 & 0.469 \\
\hline & $<50$ & 46 & $0.91 \pm 0.51$ & & \\
\hline \multirow[t]{2}{*}{ Gender } & Male & 72 & $0.88 \pm 0.50$ & 0.392 & 0.696 \\
\hline & Female & 17 & $0.83 \pm 0.53$ & & \\
\hline \multirow[t]{3}{*}{ Differentiation } & Well & 6 & $0.88 \pm 0.40$ & $F=0.531^{\mathrm{a}}$ & 0.590 \\
\hline & Moderately & 57 & $0.91 \pm 0.52$ & & \\
\hline & Poorly & 26 & $0.79 \pm 0.48$ & & \\
\hline \multirow{2}{*}{$\begin{array}{l}\text { Clinical TNM } \\
\text { stage }\end{array}$} & | to || & 19 & $1.17 \pm 0.58$ & 3.106 & 0.003 \\
\hline & III to IV & 70 & $0.79 \pm 0.45$ & & \\
\hline \multirow[t]{2}{*}{ Metastasis } & Yes & 46 & $0.74 \pm 0.51$ & -2.537 & 0.013 \\
\hline & No & 43 & $1.00 \pm 0.46$ & & \\
\hline \multirow[t]{2}{*}{ With cirrhosis } & Yes & 43 & $0.86 \pm 0.52$ & -0.143 & 0.886 \\
\hline & No & 46 & $0.88 \pm 0.48$ & & \\
\hline \multirow[t]{2}{*}{ AFP $(\mu \mathrm{g} / \mathrm{L})$} & $\geq 400$ & 35 & $0.90 \pm 0.51$ & -0.015 & 0.988 \\
\hline & $<400$ & 38 & $0.90 \pm 0.49$ & & \\
\hline \multirow{2}{*}{$\begin{array}{l}\text { Portal vein tumor } \\
\text { embolus }\end{array}$} & Yes & 29 & $0.76 \pm 0.53$ & -1.400 & 0.165 \\
\hline & No & 60 & $0.92 \pm 0.48$ & & \\
\hline \multirow[t]{2}{*}{$\begin{array}{l}\text { Tumor capsular } \\
\text { infiltration }\end{array}$} & $\begin{array}{l}\text { No capsular } \\
\text { or capsular } \\
\text { infiltration }\end{array}$ & 48 & $0.76 \pm 0.49$ & -2.308 & 0.023 \\
\hline & $\begin{array}{l}\text { No capsular } \\
\text { infiltration }\end{array}$ & 41 & $1.00 \pm 0.49$ & & \\
\hline \multirow[t]{2}{*}{ Tumor nodes } & Multiple & 40 & $0.75 \pm 0.47$ & -2.015 & 0.047 \\
\hline & Single & 49 & $0.96 \pm 0.51$ & & \\
\hline \multirow{2}{*}{$\begin{array}{l}\text { Tumor diameter } \\
(\mathrm{cm})\end{array}$} & $\geq 5$ & 73 & $0.91 \pm 0.51$ & 1.515 & 0.133 \\
\hline & $<5$ & 15 & $0.70 \pm 0.46$ & & \\
\hline \multirow[t]{2}{*}{ Vaso-invasion } & Yes & 32 & $0.79 \pm 0.53$ & -1.116 & 0.267 \\
\hline & No & 56 & $0.92 \pm 0.49$ & & \\
\hline \multirow[t]{2}{*}{ HBV infection } & Yes & 73 & $0.84 \pm 0.51$ & -1.347 & 0.181 \\
\hline & No & 16 & $1.02 \pm 0.45$ & & \\
\hline \multirow[t]{2}{*}{ HCV infection } & Yes & 29 & $0.94 \pm 0.51$ & 0.912 & 0.364 \\
\hline & No & 60 & $0.84 \pm 0.50$ & & \\
\hline
\end{tabular}

$\Delta$ Paired $t$-student test was performed.

${ }^{a}$ One-way analysis of variance (ANOVA) test was performed.

characteristic curve (ROC) was employed to identify the diagnostic value. The relationship between miR-148a and recurrence was analyzed by using the Kaplan-Meier survival method. Statistical significance was determined at a $P<0.05$ level. 


\section{Results}

Significantly lower expression of miR-148a in the HCC tissues was detected than that in the adjacent noncancerous hepatic tissues (Table 1, Figure 1). Furthermore, the ROC curve was performed to identify the diagnostic value of miR-148a level in HCC. The area under the curve (AUC) of miR-148a was 0.761 (95\% CI 0.692 to $0.830, P<0.001)$. The cut-off value for miR-148a was the median $2^{-\Delta \mathrm{cq}} 0.87$. The sensitivity and specificity were $76.3 \%$ and $50.6 \%$, respectively (Figure 2 ). With regard to clinical TNM stages, miR-148a expression in early stages (I and II) was remarkably higher than that in advanced stages (III and IV). Lower levels of miR-148a were found in HCC patients with metastasis, without capsular or with capsular infiltration and multiple tumor nodes, in comparison with patients of corresponding traits. In addition, according to Spearman's correlation, negative correlations were found between miR-148a expression and several clinicopathological parameters, including TNM stages, metastasis and the status of capsular infiltration. However, there was no association between miR148a expression and other clinicopathological features, for instance, age, histological differentiation grades, cirrhosis, plasma alpha-fetoprotein (AFP) concentrations, $\mathrm{HBV}, \mathrm{HCV}$, vaso-invasion, portal vein tumor embolus or tumor size. Sixty-one among 76 patients were followed up and time-to-recurrence was collected. Time-torecurrence for all 61 cases was $57.84 \pm 3.03$ weeks. The patients with high expression of miR-148a (higher than the median level) had a longer time-to-recurrence in comparison to those with low expression $(61.47 \pm 3.45$ versus $50.56 \pm 4.15)$, however, the difference was not significant $(P=0.238$, Figure 1$)$. Additionally, we performed the univariate analysis and results showed that miR-148a, as well as other parameters, was not a predictor for the recurrence of HCC in the current study (data not shown).

\section{Discussion}

Most recently, miR-148a expression was reported by Gailhouste et al. to be frequently down-regulated in mouse and human HCC cell lines, as well as in biopsies of HCC patients [25]. Concurrently, consistent decreased expression of miR-148a in HCC tissues was found by Zhang et al. [24], as compared with normal livers. Both of the aforementioned studies used normal liver tissues as controls. Gailhouste et al. [25] found that miR-148a expression was reduced by more than 5 -fold in HCC biopsies, relative to the normal liver group (median, 0.293 and 1.674, respectively). In the present study, accordant underexpression of miR-148a in HCC tissues was also observed, in comparison with the corresponding adjacent liver tissues in the same patients. Furthermore, the ROC curve indicates a moderate diagnostic value of miR-148a in HCC with the AUC as 0.761 . Besides, lower expression of miR-148a was also detected in several HCC cell lines (HepG2, HepB3, SNU449 and SMMC7211), than the normal hepatic cell line LO2 (data not shown). The results of our study, together with those reported previously [24] [25], indicate that miR-148a plays a critical role as a tumor suppressor miRNA in hepatocarcinogenesis. However, the average fold change of miR-148a level varied in comparison to Gailhouste et al. [25] (1.655 versus 5.713). The different controls used by Gailhouste et al. [25] and our group (healthy liver tissues versus corresponding noncancerous liver tissues) may partially explain the disparity. It might be that the miR-148a expression is lower in noncancerous liver tissue of HCC patients than in liver tissue of healthy controls. It might also be of interest to investigate the dynamic change of miR-148a expression in the hepatocarcinogenesis and progression of HCC. For instance, a comparison of the miR-148a levels in normal liver, cirrhotic tissue, adjacent noncancerous liver, hepatic adenoma, atypical hyperplasia, and HCC tissues would be worthwhile exploring.

MiRNAs can be identified in serum and plasma in an extraordinarily stable form, which leads to the possibility to determine the expression of miRNAs in blood samples $[30,31]$. The serum level of miR-148a was detected in colorectal cancer and could be regarded as a marker to predict early tumor recurrence [32]. miR148 a was determined in the serum of other malignancies, such as breast cancer, gastric cancer and multiple myeloma [33-35]. Gailhouste et al. [25] also assessed the value of circulating miR-148a as a noninvasive HCC biomarker in blood serum in HCC. However, only 11 cases with $\mathrm{HCV}$ infection were included. The significance of circulating miR-148a in the early diagnosis and prognosis prediction of HCC remains unclarified. Further studies will be required to explore the alteration of miR-148a expression in serum and in tissue, as well as to investigate the relationship between serum miR-148a level and the clinicopathological parameters of HCC patients.

Concerning the relationship between miR-148a expression and clinicopathological parameters, Yan et al. [36] determined lower expression of miR-148a in 17 cases of poorly-differentiated HCC tissues relative to 15 cases of well-differentiated HCC tissues by using an miRNA microarray analysis. In the current study, similar trend was observed. In the poorly-differentiated group, the relevant miR-148a level was $0.79 \pm 0.48$, slightly lower than that in well-differentiated $(0.88 \pm 0.4)$ and moderately-differentiated $(0.9 \pm 0.52)$ groups. However, the differences did not reach a statistically significant level. To our knowledge, no study has reported the relationship between miR-148a expression and the clinical TNM stages of HCC. In the present study, for the 


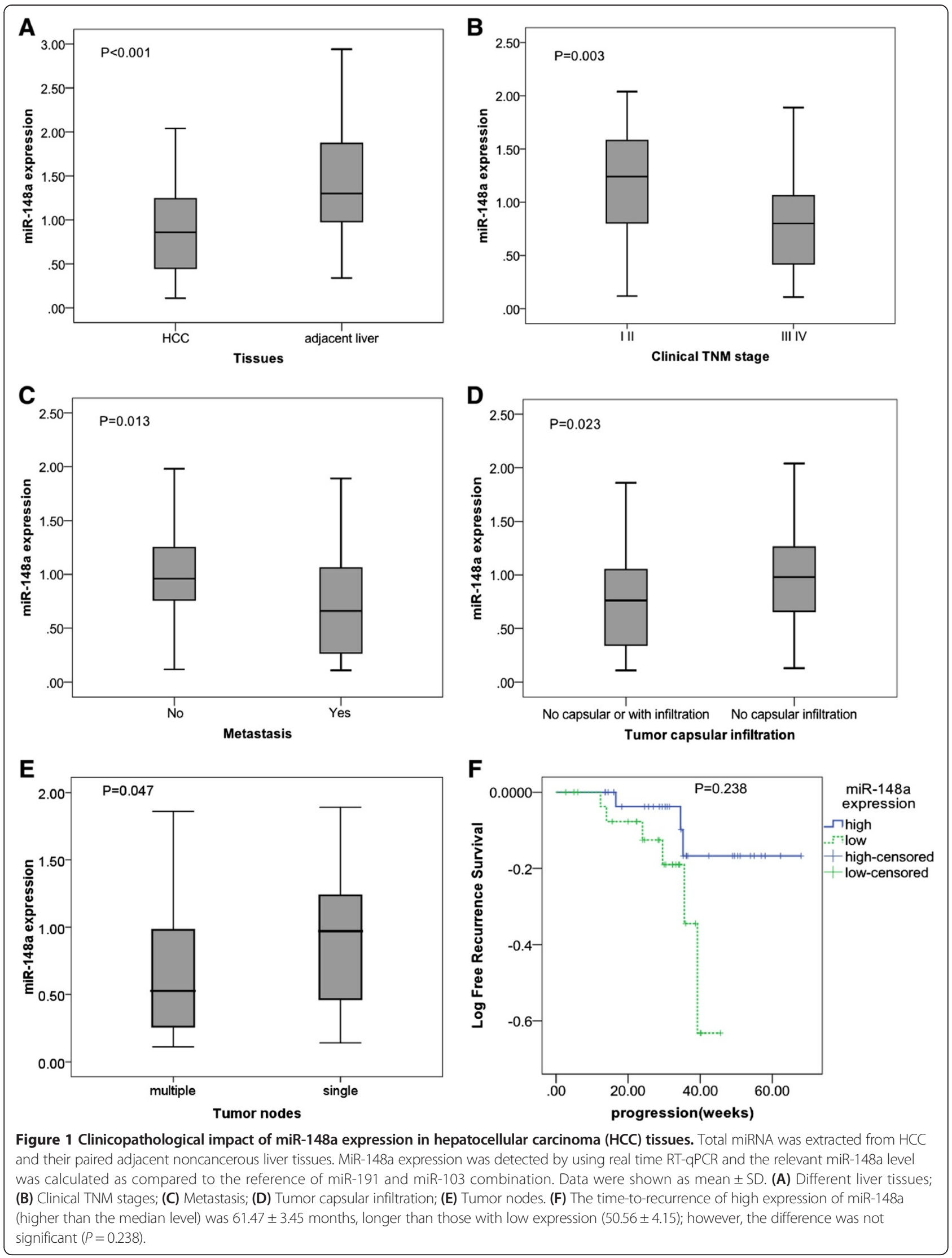




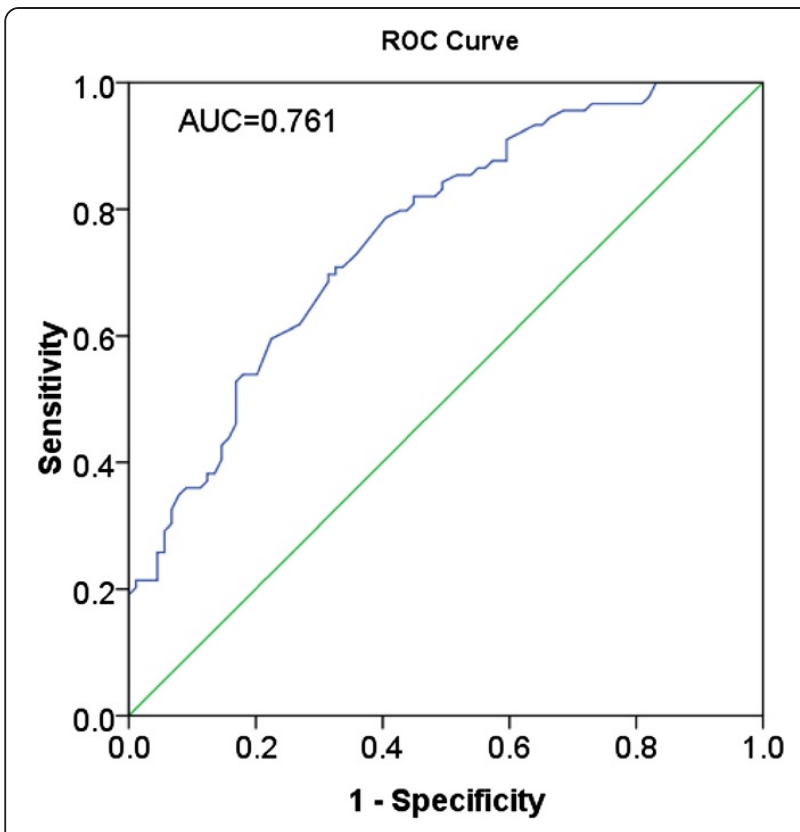

Figure 2 Receiver operator characteristic (ROC) curve of miR-148a level in hepatocellular carcinoma (HCC). The area under the curve (AUC) of miR-148a was 0.761 (95\% Cl 0.692 to $0.830, P<0.001)$.

first time, we have found that miR-148a expression in stages III and IV was lower than that in stages I and II. Furthermore, miR-148a expression was down-regulated in the metastatic group compared with that in the nonmetastatic group. Additionally, miR-148a expression was correlated with the status of tumor cell infiltration into the capsule and the numbers of tumor nodes. The status of tumor cell infiltration and tumor nodes generally reflects tumor invasion and metastasis and disease deterioration. Zhang et al. [24] reported that miR-148a decreased significantly in those HCC samples with portal vein tumor thrombus. In this study, we also found that in the subgroup with portal vein tumor embolus, the miR-148a level $(0.76 \pm 0.53)$ was lower than that in the subgroup without portal vein tumor embolus $(0.92$ \pm 0.48 ). Despite the fact that no statistically significant association was found between miR-148a and portal vein tumor embolus, the results from Zhang et al. [24] and this current study point in the same direction, that is that there is a noticeable relationship between miR148a and the infiltration of tumor cells, migration, invasion and metastasis of HCC. Hence, it may be valuable to clinically examine miR-148a expression for the prediction of metastasis and deterioration of HCC. Next, we also investigated the relationship between miR-148a level and recurrence. The HCC patients with high expression of miR-148a had a longer time-to-recurrence than those with low expression $(61.47 \pm 3.45$ versus $50.56 \pm 4.15)$; however, the difference is not significant.
A larger cohort is needed to determine the correlation between miR-148a and tumor recurrence in patients in the future.

The mechanisms whereby miR-148a was reduced in the advanced stages of HCC could be related to diverse target genes and pathways involved. The epithelialmesenchymal transition (EMT) was reported to be suppressed by miR-148a via targeting Met/Snail signaling [24]. Yan et al. [36] further revealed that miR-148a inhibits the metastasis of HCC by blocking EMT and cancer stem cells (CSCs)-like properties through effects on the Wnt signaling. Both Gailhouste et al. [25] and Long et al. [37] discovered a possible miR-148aDNA methyltransferase (DNMT) 1 regulatory circuit in HCC. The aforementioned target genes and relevant pathways can help to explain the role of miR-148a on the metastasis and deterioration of HCC.

\section{Conclusions}

Together with previous reports, the current observations strongly suggest that miR-148a acts as a tumor suppressor miRNA, which plays a vital role in the tumorigenesis and deterioration of human HCC. The current finding may help to identify potential prognostic biomarkers for HCC FFPE samples and provide a promising alterative strategy for the therapeutic treatment of HCC patients.

\section{Abbreviations}

AFP: alpha-fetoprotein; ANOVA: One-way analysis of variance; AUC: area under the curve; CSCs: cancer stem cells; DNMT: DNA methyltransferase;

EMT: epithelial-mesenchymal transition; FFPE: formalin-fixed paraffin-embedded; HBV: hepatitis B virus; HCC: hepatocellular carcinoma; HCV: hepatitis C virus; miR-148a: microRNA-148a; ROC: receiver operator characteristic curve;

SD: mean \pm standard deviation.

\section{Competing interests}

The authors declare that they have no competing interests.

\section{Authors' contributions}

LP conceived, designed and performed the experiments and analyzed the data. SH analyzed the data and wrote the manuscript. $\mathrm{RH}$ analyzed the data and wrote the manuscript. MR conceived and designed the experiments and analyzed the data. YD performed the experiments and corrected the paper. GC conceived, designed and supervised the experiments and corrected the paper. All authors read and approved the final manuscript.

\section{Acknowledgements}

The study was supported partly by the Fund of Guangxi Natural Scientific Research (number 2013GXNSFBA019191), Guangxi Provincial Health Bureau Scientific Research Project (Z2013201), and the Fund of National Natural Science Foundation of China (NSFC 81360327). The funders had no role in study design, data collection and analysis or decision to prepare and publish the manuscript.

\section{Author details}

'Department of Radiotherapy, First Affiliated Hospital of Guangxi Medical University, 6 Shuangyong Road, Nanning, Guangxi Zhuang Autonomous Region 530021, PR China. 'Department of Medical Oncology, First Affiliated Hospital of Guangxi Medical University, 6 Shuangyong Road, Nanning, Guangxi Zhuang Autonomous Region 530021, PR China. ${ }^{3}$ Research Department, Affiliated Cancer Hospital, Guangxi Medical University, 71 Hedi Road, Nanning, Guangxi Zhuang Autonomous Region 530021, PR China.

${ }^{4}$ Department of Pathology, First Affiliated Hospital of Guangxi Medical 
University, 6 Shuangyong Road, Nanning, Guangxi Zhuang Autonomous Region 530021, PR China.

Received: 18 August 2014 Accepted: 18 November 2014 Published online: 02 December 2014

\section{References}

1. Liu B, Mao A, Liu D: The hypothesis of an effective strategy for resistance of hepatocellular carcinoma to therapy-autophagy. West Indian Med J 2011, 60(6):666-668.

2. Jemal A, Bray F, Center MM, Ferlay J, Ward E, Forman D: Global cancer statistics. CA Cancer J Clin 2011, 61(2):69-90.

3. Vertemati M, Moscheni C, Petrella D, Lamperti L, Cossa M, Gambacorta M, Goffredi M, Vizzotto L: Morphometric analysis of hepatocellular nodular lesions in HCV cirrhosis. Pathol Res Pract 2012, 208(4):240-244.

4. Abdo AA, Al-Ahdal MN, Khalid SS, Helmy A, Sanai FM, Alswat K, Al-Hamoudi W, Ali SM, Al-Ashgar HI, Al-Mdani A, Albenmousa A, Al Faleh FZ, Al-Anazi M, Khalaf N, Al-Qahtani A: IL28B polymorphisms predict the virological response to standard therapy in patients with chronic hepatitis $C$ virus genotype 4 infection. Hepatol Int 2013, 7(2):533-538.

5. Park CW, Cho MC, Hwang K, Ko SY, Oh HB, Lee HC: Comparison of quasispecies diversity of HCV between chronic hepatitis $C$ and hepatocellular carcinoma by ultradeep pyrosequencing. BioMed Res Int 2014, 2014:853076.

6. Liu F, You X, Chi X, Wang T, Ye L, Niu J, Zhang X: Hepatitis B virus X protein mutant $\mathrm{HBXDelta127}$ promotes proliferation of hepatoma cells through up-regulating miR-215 targeting PTPRT. Biochem Biophys Res Commun 2014, 444(2):128-134.

7. Kretzer IF, Do Livramento A, Da Cunha J, Goncalves S, Tosin I, Spada C, Treitinger A: Hepatitis C worldwide and in Brazil: silent epidemic-data on disease including incidence, transmission, prevention, and treatment. Sci World J 2014, 2014:827849.

8. Hu L, Chen G, Yu H, Qiu X: Clinicopathological significance of RASSF1A reduced expression and hypermethylation in hepatocellular carcinoma. Hepatol Int 2010, 4(1):423-432.

9. Chen G, Luo D: Expression of decoy receptor 3 in liver tissue microarrays. Natl Med J India 2008, 21(6):275-278.

10. Chen G, Dang YW, Luo DZ, Feng ZB, Tang XL: Expression of heparanase in hepatocellular carcinoma has prognostic significance: a tissue microarray study. Oncol Res 2008, 17(4):183-189.

11. Chen G, Luo DZ, Liu L, Feng ZB, Guo F, Li P: Hepatic local microenvironmental immune status in hepatocellular carcinoma and cirrhotic tissues. West Indian Med J 2006, 55(6):403-408.

12. Chen G, Luo D: Over-expression of decoy receptor 3 in gastric precancerous lesions and carcinoma. Ups J Med Sci 2008, 113(3):297-304.

13. Yang $M$, Chen $G$, Dang $Y$, Luo D: Significance of decoy receptor 3 in sera of hepatocellular carcinoma patients. Ups J Med Sci 2010, 115(4):232-237.

14. Ruan ZP, Xu R, LV Y, Tian T, Wang WJ, Guo H, Nan KJ: PTEN enhances the sensitivity of human hepatocellular carcinoma cells to sorafenib. Oncol Res 2012, 20(2-3):113-121.

15. Zhuang $L$, Wu Y, Han J, Ling $X$, Wang L, Zhu C, Fu Y: A network biology approach to discover the molecular biomarker associated with hepatocellular carcinoma. BioMed Res Int 2014, 2014:278956.

16. Sen R, Ghosal S, Das S, Balti S, Chakrabarti J: Competing endogenous RNA: the key to posttranscriptional regulation. Sci World J 2014, 2014:896206.

17. Omelia EJ, Uchimoto ML, Williams G: Quantitative PCR analysis of bloodand saliva-specific microRNA markers following solid-phase DNA extraction. Anal Biochem 2013, 435(2):120-122.

18. Hui A, How C, Ito E, Liu FF: Micro-RNAs as diagnostic or prognostic markers in human epithelial malignancies. BMC Cancer 2011, 11:500

19. Di Leva G, Briskin D, Croce CM: MicroRNA in cancer: new hopes for antineoplastic chemotherapy. Ups J Med Sci 2012, 117(2):202-216.

20. Siegrist F, Singer T, Certa U: MicroRNA expression profiling by bead array technology in human tumor cell lines treated with interferon-alpha-2a. Biol Proced Online 2009, 11:113-129.

21. Papagiannakopoulos T, Kosik KS: MicroRNAs: regulators of oncogenesis and stemness. BMC Med 2008, 6:15.

22. Sato F, Hatano E, Kitamura K, Myomoto A, Fujiwara T, Takizawa S, Tsuchiya S, Tsujimoto G, Uemoto S, Shimizu K: MicroRNA profile predicts recurrence after resection in patients with hepatocellular carcinoma within the Milan Criteria. PLoS One 2011, 6(1):e16435.
23. Hung $\mathrm{CH}$, Chiu YC, Chen $\mathrm{CH}, \mathrm{Hu} \mathrm{TH}$ : MicroRNAs in hepatocellular carcinoma: carcinogenesis, progression, and therapeutic target. BioMed Res Int 2014, 2014:486407.

24. Zhang JP, Zeng C, Xu L, Gong J, Fang JH, Zhuang SM: MicroRNA-148a suppresses the epithelial-mesenchymal transition and metastasis of hepatoma cells by targeting Met/Snail signaling. Oncogene 2014, 33(31):4069-4076.

25. Gailhouste L, Gomez-Santos L, Hagiwara K, Hatada I, Kitagawa N, Kawaharada K, Thirion M, Kosaka N, Takahashi RU, Shibata T, Miyajima A, Ochiya T: miR-148a plays a pivotal role in the liver by promoting the hepatospecific phenotype and suppressing the invasiveness of transformed cells. Hepatology 2013, 58(3):1153-1165.

26. Chen G, Umelo IA, LV S, Teugels E, Fostier K, Kronenberger P, Dewaele A, Sadones J, Geers C, De Greve J: miR-146a inhibits cell growth, cell migration and induces apoptosis in non-small cell lung cancer cells. PLoS One 2013, 8(3):e60317.

27. Dang Y, Luo D, Rong M, Chen G: Underexpression of miR-34a in hepatocellular carcinoma and its contribution towards enhancement of proliferating inhibitory effects of agents targeting c-MET. PLoS One 2013, 8(4):e61054.

28. Rong M, Chen G, Dang Y: Increased miR-221 expression in hepatocellular carcinoma tissues and its role in enhancing cell growth and inhibiting apoptosis in vitro. BMC Cancer 2013, 13:21.

29. Chen $G$, Kronenberger $P$, Teugels E, Umelo IA, De Greve J: Targeting the epidermal growth factor receptor in non-small cell lung cancer cells: the effect of combining RNA interference with tyrosine kinase inhibitors or cetuximab. BMC Med 2012, 10:28.

30. Ramshankar $\mathrm{V}$, Krishnamurthy A: Lung cancer detection by screening presenting circulating miRNAs as a promising next generation biomarker breakthrough. Asian Pac J Cancer Prev 2013, 14(4):2167-2172.

31. Qu H, Xu W, Huang Y, Yang S: Circulating miRNAs: promising biomarkers of human cancer. Asian Pac J Cancer Prev 2011, 12(5):1117-1125.

32. Tsai HL, Yang IP, Huang CW, Ma CJ, Kuo CH, Lu CY, Juo SH, Wang JY: Clinical significance of microRNA-148a in patients with early relapse of stage II stage and III colorectal cancer after curative resection. Trans/ Res 2013, 162(4):258-268.

33. Kodahl AR, Zeuthen P, Binder H, Knoop AS, Ditzel HJ: Alterations in circulating miRNA Levels following early-stage estrogen receptorpositive breast cancer resection in post-menopausal women. PLoS One 2014, 9(7):e101950

34. Kim SY, Jeon TY, Choi Cl, Kim DH, Kim DH, Kim GH, Ryu DY, Lee BE, Kim HH: Validation of circulating miRNA biomarkers for predicting lymph node metastasis in gastric cancer. J Mol Diagn 2013, 15(5):661-669.

35. Huang JJ, Yu J, Li JY, Liu YT, Zhong RQ: Circulating microRNA expression is associated with genetic subtype and survival of multiple myeloma. Med Oncol 2012, 29(4):2402-2408.

36. Yan H, Dong $X$, Zhong $X$, Ye J, Zhou Y, Yang X, Shen J, Zhang J: Inhibitions of epithelial to mesenchymal transition and cancer stem cells-like properties are involved in miR-148a-mediated anti-metastasis of hepatocellular carcinoma. Mol Carcinog 2014, 53(12):960-969.

37. Long XR, He Y, Huang C, Li J: MicroRNA-148a is silenced by hypermethylation and interacts with DNA methyltransferase 1 in hepatocellular carcinogenesis. Int J Oncol 2014, 44(6):1915-1922.

doi:10.1186/s40001-014-0068-2

Cite this article as: Pan et al:: Decreased expression and clinical significance of miR-148a in hepatocellular carcinoma tissues. European Journal of Medical Research 2014 19:68. 